\title{
EEG Analysis of the Contribution of Music Therapy and Virtual Reality to the Improvement of Cognition in Alzheimer's Disease
}

\author{
Alexie Byrns ${ }^{1}$, Hamdi Ben Abdessalem¹, Marc Cuesta ${ }^{2}$, Marie-Andrée Bruneau², \\ Sylvie Belleville ${ }^{2}$, Claude Frasson ${ }^{1}$ \\ ${ }^{1}$ Département d'Informatique et de Recherche Opérationnelle, Université de Montréal, Montréal, Canada; ${ }^{2}$ Centre de \\ Recherche de l'Institut de Gériatrie de Montréal, Montréal, Canada
}

Correspondence to: Alexie Byrns, marc.cuesta@criugm.qc.ca; Marie-Andrée Bruneau, frasson@iro.umontreal.ca Keywords: Alzheimer's Disease, Brain Assessment, Virtual Reality, Music Therapy, Neurofeedback, Memory, Cognition

Received: June 12, $2020 \quad$ Accepted: August 21, $2020 \quad$ Published: August 24, 2020

Copyright $\odot 2020$ by author(s) and Scientific Research Publishing Inc.

This work is licensed under the Creative Commons Attribution International License (CC BY 4.0).

http://creativecommons.org/licenses/by/4.0/

\section{(c) (1) Open Access}

\section{ABSTRACT}

\begin{abstract}
Alzheimer's disease is the most common form of dementia, affecting nearly 9.9 million new people every year. The disease provokes important memory and cognitive impairment, eventually causing individuals to forget their loved ones and rendering them completely dependent on their caretakers. Alzheimer's patients typically experience more negative emotions, such as frustration and apathy, than healthy older adults. There is currently no cure for the disease. Our research group explores how the integration of virtual reality (VR) and an EEG-based intelligent agent in music therapy can alleviate psychological and cognitive symptoms of the disease. We propose a theory explaining how, through activation of the brain reward system, music can reduce negative emotions, increase positive emotions and as a result increase performance on cognitive tasks. The results of our experimental study concord with our theory: emotional states of participants are improved, as per recorded through EEG, and performances on memory tasks show improvement following the intervention. We believe that the combination of EEG brain assessment, VR and music therapy is a promising method for emotional states and cognitive symptoms of Alzheimer's disease.
\end{abstract}

\section{INTRODUCTION}

Elvis Presley, Céline Dion, The Beatles and so many others have contributed to our lives in one way or another, be it by shaping society or our simply by serenading us to work on the radio. Even though it 
serves no apparent evolutionary purpose or means to survival, music has persisted through time in every known culture [1]. Music no doubt is an integral part of our lives. In addition to music being a ubiquitous art form and cultural activity, it contributes to both our emotional and physical well-being in more ways than one. Evidence suggests that music can have a positive effect on a variety of diseases and disorders, including Alzheimer's disease (AD) [2,3].

$\mathrm{AD}$ is the most common form of dementia and is characterized by memory and cognitive impairments. Memory is the first to be affected, where individuals often report having more difficulty remembering certain things than usual. Alzheimer's disease is progressive and its earliest phase is characterized by a state of subjective cognitive decline (SCD). While some persons with SCD stagnate, persons with SCD are at a greater risk of progressing to the disease.

No cure has yet been found, though some pharmacological interventions may reduce the symptoms. As patients progress through later stages of the disease, apathy-the lack of motivation-settles in, and patients become seemingly emotionally unresponsive [4]. This emotional state may be a hinderance to the already impaired cognitive state of patients. Indeed, a recent study showed that decreasing negative emotions in $\mathrm{AD}$ patients promotes better cognitive performance in both memory and non-memory related tasks $[5,6]$. We believe that by intervening at the emotional level, it is possible to observe a positive influence on cognitive performances including memory tasks in AD patients. We base this emotional intervention on the integration of music therapy in virtual reality (VR), as both approaches have already proven to increase emotional well-being in $\mathrm{AD}$ patients $[7,8]$.

Many studies have investigated the relationship between music and the emotional response it generates. Positron emission tomography (PET) and functional magnetic resonance imaging (fMRI) studies have identified correlations between the music-induced sensation of pleasure and the activity of specific brain structures, including the ventral striatum, midbrain, amygdala, orbitofrontal cortex and the medial prefrontal cortex [9]. These regions are part of the brain reward system (BRS) circuitry, a network of interconnected brain structures which play a role in the sensation of pleasure and reward [10]. It is suggested that music retains its emotional influence through the recruitment of the BRS.

In this paper we present a theory by which music associated with VR could enhance cognitive functions in $\mathrm{AD}$ patients. We support this view through an experimental study we conducted using electroencephalography (EGG) recordings of participant's brain activity. We interpret emotional responses of the subjects through EEG measures.

The rest of this paper is organized as follows. The first section gives an overview of AD. The second section explores evidence proving music therapy's benefits for AD patients. The third section focuses on the BRS and its role with music, where we propose a new theory explaining how music could indirectly enhance memory and other cognitive functions in AD patients. The fourth section explains the importance of EEG measurements and how they can be used to track emotional responses. The following section presents the results of our study carried out on SCD patients involving music therapy, to provide support to our theory. We conclude our paper with a discussion about the link between music and cognition.

\section{ALZHEIMER'S DISEASE}

Alzheimer's disease is a debilitating disease affecting memory and cognitive abilities. Most people who suffer from the disease display their first clinical symptoms after the age of 70 [11]. Although some cases can be tied to genetic mutations-often the early-onset familial form-most cases are sporadic, and their cause is unknown.

The progression of the disease manifests itself through many changes at a cognitive, behavioral and psychological level. The disease can start with subjective cognitive decline (SCD), where the patient notices impairment of their memory, but it cannot be detected with formal tests and does not affect their day to day lives. Some people remain in this state for decades and never progress to AD. People who experience SCD are more at risk of progressing towards the disease than healthy older adults. Those who do progress to $\mathrm{AD}$ experience gradual loss of memory and cognitive abilities, characterized among other things by dif- 
ficulties in problem solving, communication and visuospatial perception. As they reach the later stages of the disease, patients become entirely dependent of their caregivers, apathetic and bedridden [12].

In addition to cognitive impairment, $\mathrm{AD}$ patients experience diminished psychological states. Among the many symptoms of the disease, depression and apathy are arguably the most common $[4,13,14]$. Studies show that $\mathrm{AD}$ patients often become anxious and uninterested in things that used to bring them joy [4]. Regularly having a negative emotional state is a common in $\mathrm{AD}$ patients [15], and this may aggravate the already-impaired cognitive state of patients.

On a pathophysiological level, $\mathrm{AD}$ is characterized by three major abnormalities: 1) brain atrophy of specific regions, 2) extracellular amyloid plaques, and 3) neurofibrillary tangles (NFT). AD brains reveal substantial neuronal death. The gyri and sulci which give the brain its distinct shriveled appearance are narrowed and widened, respectively. The ventricles also suffer from this neuronal death, becoming much larger than those of a healthy brain. At the extracellular level, $\mathrm{A} \beta$ amyloid plaques are found surrounding the neurons. These deposits are formed by the aggregation of amyloid $\beta$ proteolytic fragments and cause disruptions in cell-cell communication. At the intracellular level, neurons display cytoskeleton abnormalities, most notably the accumulation of NFTs. These are the result of abnormal proteins which, when in their healthy form, contribute to intracellular transport, especially in the axons [16]. By bringing about disturbances in the axonal transport, tangles impair synaptic stability and eventually cause the death of the neuron [11].

Many studies have been conducted in hopes of curing the disease. Although no research has been successful, these years of research have helped better understand the disease, bringing us closer to understanding its earliest phases. While many research groups still focus on potential pharmacological interventions, a growing number of studies have begun to investigate the potential of non-pharmacological interventions in the reduction of cognitive decline and other symptoms of the disease. These interventions, which range from visual arts and music, to physical activity and electromagnetic stimulation, have proven to be promising avenues. Either on their own or by combining them with pharmacological interventions, non-pharmacological interventions are worth further investigating. In this line of thought, we explore the potential of music therapy for SCD patients.

\section{MUSIC THERAPY FOR ALZHEIMER'S DISEASE}

Music is a highly salient emotional stimulus. It easily generates emotions which can cause changes in mood. While providing no evolutionary advantage or association to survival, music has persisted through time as an activity providing pleasure, either when actively created or when passively listened to. Its strong ties to the triggering of emotional responses, sometimes even of involuntary movements such as swaying to the beat [17-19], may have contributed to its persistence.

Studies show that music causes positive changes in psychological and behavioral states. AD patients are no exception to this. Indeed, music can improve overall well-being, relations with caregivers, and decrease unwanted negative symptoms of the disease such as agitation, stress, anxiety and depression [8, 20-22]. Following a music therapy session, mild-AD patients generally experience an increased state of happiness and a decreased state of sadness [23].

Music also shows great potential for neurological rehabilitation. Interestingly, musical memory is partly spared even in very late stages of the disease, while other memories have become severely impaired. In terms of non-musical-related memories, reports show slowed cognitive decline [24], improved orientation, as well as enhanced memory and other cognitive functions $[8,25,26]$ following music therapy sessions in $\mathrm{AD}$ patients.

\section{THE BRAIN REWARD SYSTEM}

The brain reward system (BRS) is a group of interconnected brain structures, which play a role in the rewarding experience of stimuli. These stimuli can be anything with a positive value, such as sex, food and music. When such a stimulus appears, the reward system is activated, activating the circuitry and in turn 
giving the subjective experience of pleasure and reward [27].

The BRS is composed of structures connecting the midbrain, limbic system and prefrontal cortex. Key structures of this network include the ventral tegmental area (VTA), amygdala, and nucleus accumbens. Within its circuitry, dopamine is the main neurotransmitter, where it is both synthesized-largely by the VTA and released. Other types of neurons, such as inhibitory neurons, play a key role in the reward circuitry by regulating neuronal activity. Incorrect regulation of the pathway can result in certain diseases and disorders, such as depression, schizophrenia, addiction and Parkinson's disease [28].

The BRS plays an important role in the processing of emotional stimuli and certain memory functions. Lesions of the amygdala, a structure of this network, have shown to impair implicit memory: a type memory that stores knowledge unconsciously acquired. Lesions of the amygdala can therefore cause impairments in the unconscious recall of both perceptual and motor skills [11].

As mentioned earlier, BRS-activating stimuli can be many things. Indeed, music has repeatedly shown to activate structures related to biological reward [3, 29-31]. It is proposed [32] that music activates the BRS through interaction between cortical networks related to audition and to reward.

Activation of the reward system through music has been shown to maximize pleasure by increased activation in of specific brain structures and decreased activity in structures associated with negative emotions through inhibitory activity towards other specific structures [9]. In addition to its ties with emotional state, some studies link the BRS to certain cognitive functions. Notably, a study showed that short exposure to rewarding stimuli reduced cortisol, a so-called stress hormone, and resulted in better problem-solving performances in stressful situations [33]. Other studies also show that lesions of the amygdala have led to impaired implicit memory [11], suggesting that the BRS plays a role in the recollection of implicit memories.

Considering these previous findings, we propose the following theory: By increasing positive emotions and reducing negative emotions through the activation of the BRS, music improves cognitive functions of SCD participants. We divide this hypothesis in two research questions: 1) Does virtual music therapy increase positive emotions and decrease negative emotions? and 2) Does the virtual music therapy increase cognitive functions?

\section{OUR EXPERIMENT: MUSIC THERAPY VIRTUAL ENVIRONMENT}

\subsection{Brain Assessment: Analysis through EEG}

In the brain assessment field, many studies use physiological sensing approaches such as electroencephalography (EEG) to detect and analyze emotional states. By being readily available, inexpensive and non-invasive, EEG is a simple and easy way to get an inside view of subjects' brain activity. Through the analysis of the electrical activity emitted from the brain, researchers can better understand the inner workings of the brain.

Using EEG has not only proven to be beneficial for research, but has also showed to benefit participants themselves. A study showed that by using the assessment of engagement and frustration, appropriate help strategies could be provided to participants engaged in a physics-related game [34]. Furthermore, it has been shown that a positive mental state promotes better learning in students. Taking this into account, Chaouachi et al. [35] developed a system based on EEG-recorded engagement and workload to help keep students in a positive mental state.

By using EEG recordings, we aim to get an inside view of our participants' emotions. Using the real-time-provided emotional state, we can create an intervention better adapted to the participant. EEG headsets have shown to be well tolerated by SCD subjects $[5,6]$.

In our study, we use the Emotiv Epoc EEG headset to track emotions. The headset contains 14 electrodes spatially organized according to the International $10-20$ system, moist with a saline solution. The electrodes are placed in antero-frontal (AF3, AF4, F3, F4, F7, F8), fronto-central (FC5, FC6), parietal (P7, $\mathrm{P} 8)$, temporal $(\mathrm{T} 7, \mathrm{~T} 8)$ and occipital $(\mathrm{O} 1, \mathrm{O} 2)$ regions with two additional reference sensors placed behind the ears. The detailed position of the measured regions is shown in Figure 1. 


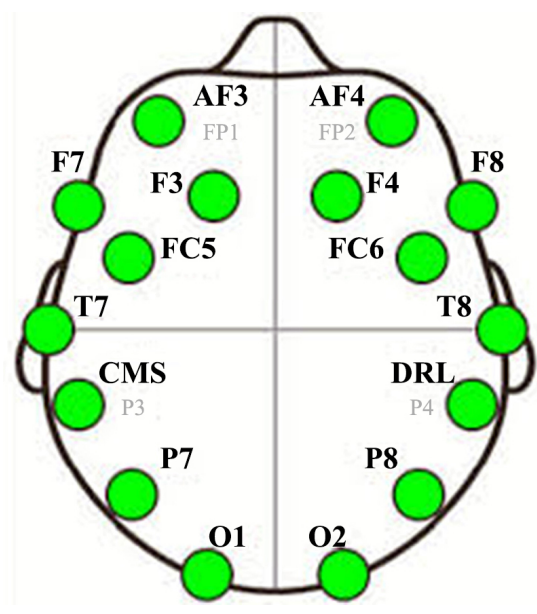

Figure 1. Emotiv headset sensors placement.

The Emotiv system generates raw EEG data (in $\mu \mathrm{V}$ ) with a $128 \mathrm{~Hz}$ sampling rate as well as the five well-known frequency bands, namely Theta (4 to $8 \mathrm{~Hz}$ ) Alpha ( 8 to $12 \mathrm{~Hz}$ ), low Beta (12 to $16 \mathrm{~Hz}$ ), high Beta ( 16 to $25 \mathrm{~Hz}$ ) and Gamma ( 25 to $45 \mathrm{~Hz}$ ). Furthermore, the system uses internal algorithms to measure the following mental states: meditation, frustration, engagement, excitement and valence.

Although we don't have access to the system's proprietary algorithms to infer these mental states, a number of studies have provided evidence showing the reliability of its output [36].

\subsection{The Virtual Environment}

Our music therapy was designed within a virtual environment. This modality was chosen based on three main factors. For one, the application of VR in a wide range of psychiatric disorders has shown its potential and proven its usefulness [37]. Studies using VR on AD patients have shown that participants tolerated well the headsets, and even enjoyed the VR experience [5]. Secondly, the virtual environment enables for a stronger feeling of immersion and increases ecological validity. This in turn provides a stronger influence of the environment on emotional state. Finally, by pairing the virtual environment with an EEG headset and integrating an intelligent agent, the environment can evolve and adapt itself as a function of the participant's EEG readings. In an attempt to improve and optimize the utilization of VR, we propose the integration of an intelligent agent capable of modifying the environment appropriately as a function of emotions.

We designed an environment resembling a theatre using Unity 3D software. We virtually placed the participant in the center of the room, on a seat, where they could easily see the stage up front and dynamically rotate their head to visually explore the room. At the start of the session, cliché red curtains opened the stage area.

A series of eight 30 second song excerpts are sequentially presented in the following order: 1) Ave Maria by Franz Schubert, 2) Eine Klein Nachtumusik: Allegro by Mozart, 3) Ukulele by Bensound, 4) Clair De Lune by Debussy, 5) La Vie En Rose by Edith Piaf, 6) Everyday by Buddy Holly, 7) La Bamba by Ritchie Valens, and finally 8) What A Wonderful World by Louis Armstrong. Each song excerpt was clearly separated from the previous by the red curtains closing and opening. The choice of music was based on studies and theories. A portion of the songs were chosen because they contained melodies with structural features associated with anxiety reduction (i.e. slow tempo, low pitches) [38]. Another portion of the songs was selected based on their popularity in North America during the years corresponding to our participant's reminiscence bump. This refers to a time period where events and memories are more likely to be remembered. The reminiscence bump corresponds to the time between the ages of 10 and 30, with the likelihood of remembering events peaking around the age of 20 years old [39]. Given that the mean age of our participants was 72, some songs we selected reached the billboard charts between the years 1957 and 
1977.

For each song, the stage displayed a selection corresponding instruments, each slightly animated, as well as firework-like visual effects (see Figure 2). The intensity of the light as well as the colors of the visual effects was chosen as a function of the emotional purpose of the song. Scenes were designed with specific shades of red, purple, blue, green and yellow, as these are suggested to be the most pleasant and arousing hues [40].

Once the eight song excerpts were done, the environment was adapted to provide the most beneficial therapeutic experience to each individual participant. This was calculated based on the participants' emotional response analyzed though EEG recordings. Indeed, different songs and different visual effects can have varying impacts on individuals.

In order to best suit each participant, we used a method with a neurofeedback agent: Neurofeedback is a type of biofeedback that measures brain waves to produce a signal that can be used as feedback. When the measured activity is cerebral activity, biofeedback is called neurofeedback [41]. The neurofeedback agent tracks the emotions of the participants while they listen to the eight songs, detects the song which provokes the best emotional impact and plays this song once again for a longer period of time. Using this neurofeedback approach, we finally adapt the playlist to the user in order to favorize the song which has the most potential effect on the participant. This means the song that has less negative effect and most positive effect on the participant's emotional state. The neurofeedback aspect of our environment enables to optimize the emotional impact for every individual participant.

\subsection{Experiments}

In order to study the impact of the music therapy environment on attention and memory performances, we created 6 attention and memory exercises using Unity 3D software. The following provides brief descriptions of each exercise.

\subsubsection{Attention Exercises}

For the first attention exercise, the participant hears a series of numbers and is invited to replicate the sequence in the same order as presented using a numerical pad. Then, another series of numbers is presented but the participants are now invited to report numbers in the reversed order. Figure 3 illustrates how the participant uses the numerical pad in order to interact with the exercise.

For the second attention exercise, participants hear a series of letters (one letter per second) and are required to click on the space bar of the keyboard each time they hear the letter " $\mathrm{A}$ ".

Finally, in the third attention exercise, participants are shown pictures of different items for a short period of time. Then, after each picture, four letters are presented and they are asked to select the letter corresponding to the first letter of the presented item's name.

\subsubsection{Memory Exercises}

For the first memory exercise, we present several objects visually or aurally to the participants and ask them to memorize them. After that, we present a series of objects and the participants are asked to select whether the object was seen, heard or never presented. Figure 4 shows the case where the participant answers they have seen the image of the car.
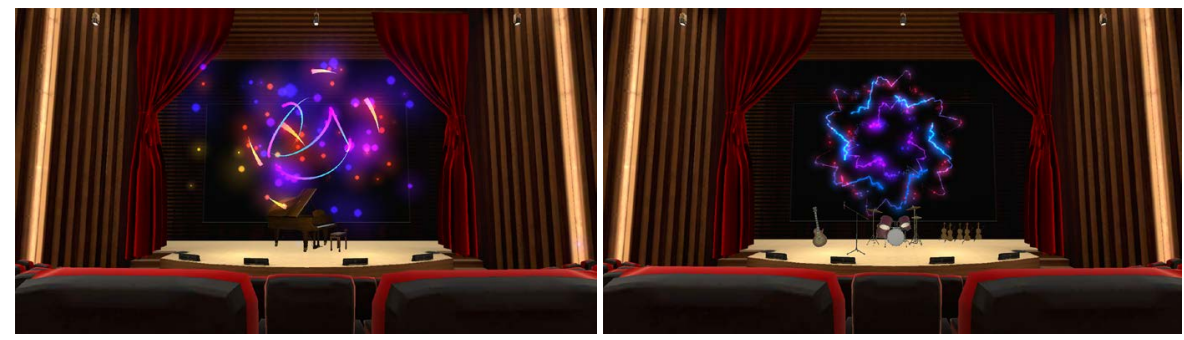

Figure 2. Scenes for two different songs from the virtual environment. 


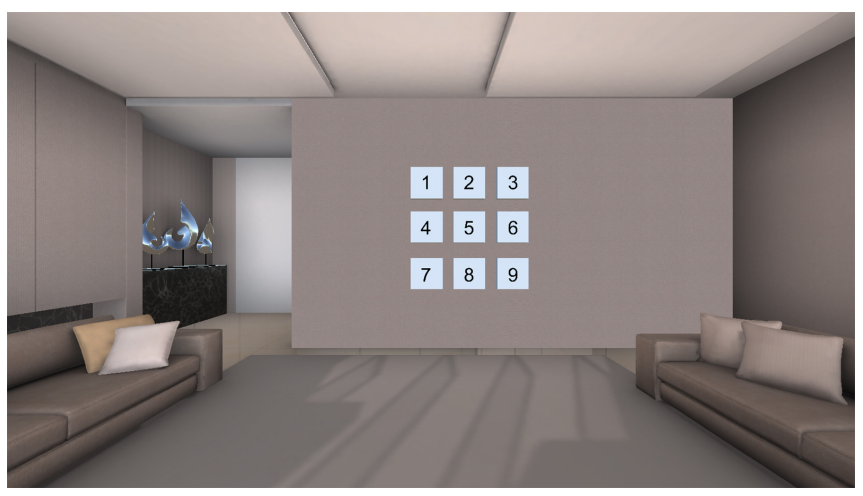

Figure 3. Screenshot of attention exercise 1.

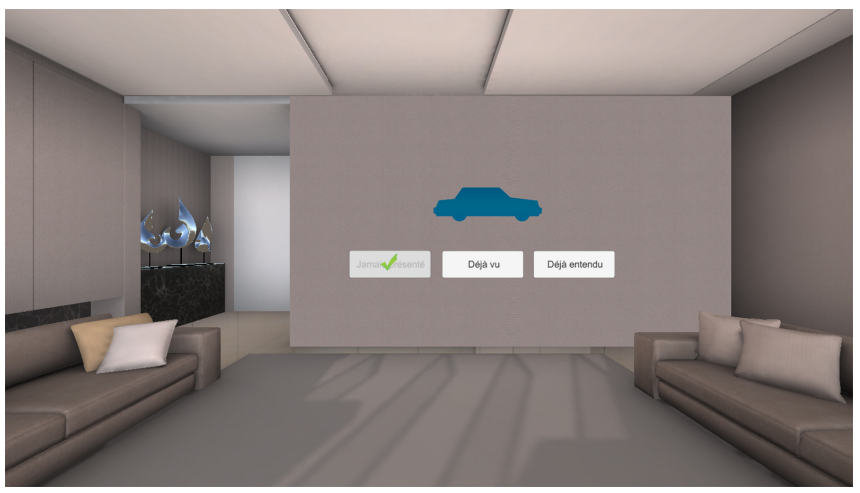

Figure 4. Screenshot of memory exercise 1.

For the second memory exercise, we present several circles spread out against the virtual wall. Then, a series of circles is highlighted one by one in order to create a sequence. The participants are invited to memorize and repeat the same sequence.

Finally, in the third memory exercise, we ask the participants to memorize sets of three pictures for a short period of time. Then, we present four sets of three similar pictures to the participants, and they are asked to select the set matching the one that was previously presented.

\subsubsection{Experimental Process}

We tested our approach on 19 participants (13 females) with subjective cognitive decline (SCD) and a mean age $=72.26(S D=5.82)$. In order to ensure that the participants were eligible for the study, they had to pass through a pre-experimental session. In this session, participants were presented with oral and written descriptions of the study and were invited to sign a consent form. Then, they performed the clinical tests necessary to confirm diagnosis of SCD and characterize them. Only the eligible participants were invited to the experimental session.

In the experimental session, participants were first invited to fill the following questionnaires: The Positive and Negative Affect Schedule (PANAS) scale [42], a self-assessment of emotions, and a questionnaire on cyber-sickness [43]. Then, we equipped the participants with an EEG headset and invited them to start solving the attention and memory exercises. Following these tests, participants were equipped with a FOVE VR headset, and the VR music therapy environment was launched. The relaxation environment lasted for about 10 minutes. Following the virtual environment, participants completed different variants of the same attention and memory exercises. Finally, the experimental session came to an end after asking the participants to once again fill the PANAS scale, cyber-sickness, AttrakDiff 2 [44] and a self-report form about the environment. Figure 5 shows the steps of the experimental process. 


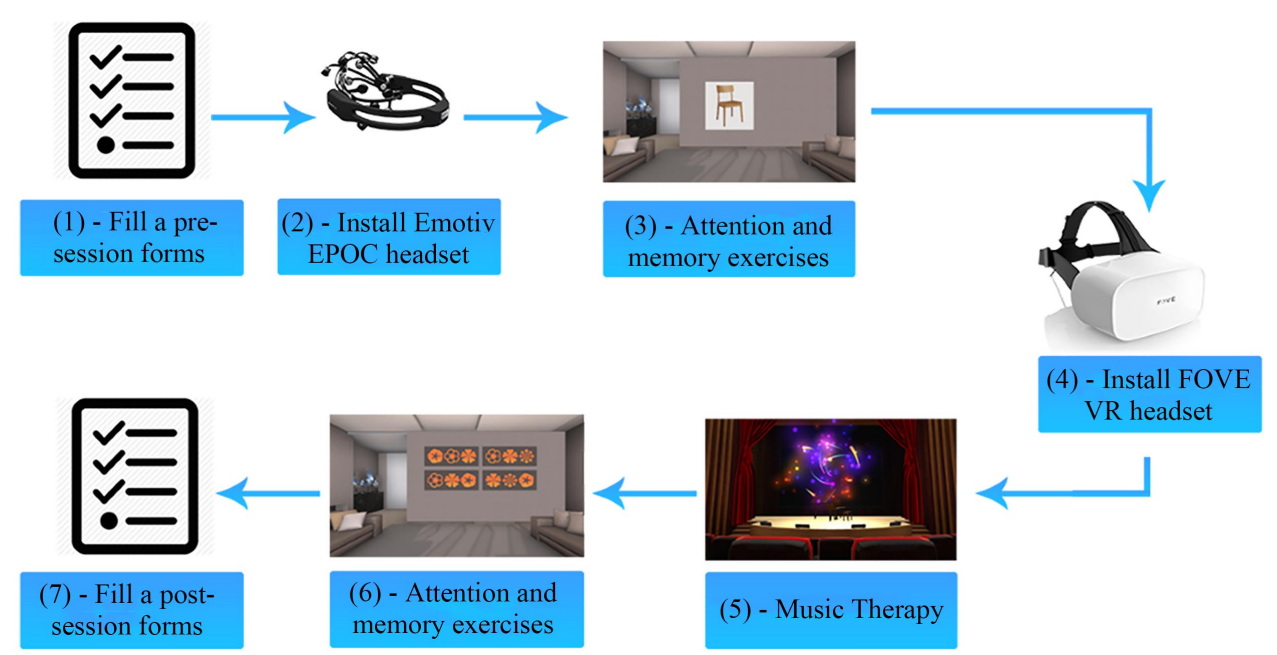

Figure 5. Process of the experiment.

\section{RESULTS}

Our first research question asked: Does virtual music therapy increase positive emotions and decrease negative emotions? To this end, we started by analyzing the emotions of the participants before, during and after the virtual music therapy immersion. This was done on the measure of frustration and valence extracted from the Emotiv EEG recordings. Results show that the mean frustration level before the music therapy was 0.69 ( 0.37 minimum and 1.00 maximum). The mean frustration level during the immersion was 0.45 ( 0.21 minimum and 0.82 maximum). After the immersion, the mean frustration level was 0.51 (minimum 0.23 and maximum 0.92). Figure 6 shows a boxplot of the mean frustration before, during and after the music therapy session.

Overall, the frustration decreased when the participants were in the virtual environment, and the positive effect on the frustration level was still observed after the music therapy.

Next, we analyzed the mean valence before, during and after the music therapy immersion. Results show that the mean valence before was 0.59 ( 0.48 minimum and 0.78 maximum). The mean valence during the immersion was 0.54 (minimum 0.47 and maximum 0.65). After, the mean valence level was 0.75 (minimum 0.55 and maximum 0.92). Figure 7 shows a boxplot of the mean valence before, during and after the virtual environment.

Overall, the valence decreased slightly when the participants were in the virtual environment but increased considerably following their immersion. We believe this is a result of the experiment's design, where the emotional optimization (choice of the song with the best emotional impact) took place at the very end of the music therapy session.

In addition, post-session appreciation forms revealed that $89.5 \%$ of the participants found that the environment was immersive while 5.3\% reported that the environment was not immersive, and the rest gave a neutral response. Results also showed that $84.2 \%$ of the participants reported that the virtual reality had a positive impact on their user experience, 5.3\% reported that the virtual reality had a negative impact and the rest gave a neutral response. Finally, $89.5 \%$ of the participants reported that they liked the environment and they found it relaxing, 5.3\% reported that it was not relaxing, and the rest gave a neutral response.

Thus, the music therapy environment reduced negative emotions, such as frustration, and increased positive emotions, like valence and relaxation. This observation led to our second research question: Does the virtual music therapy increase cognitive functions? To this end, we analyzed performance improvements on the attention and memory exercises of each participant. We begin by presenting the results of the attention exercises and follow with those of the memory exercises.

For the attention exercises, results show small improvements. For the first exercise, the general mean 
improvement was $6.59 \%$. For the second, there was a mean improvement of $1.91 \%$. The performance improvement on the third exercise was 3.51\%. More detailed results are shown in Figure 8, where we note that for exercise one, 6 participants showed improvement, 3 decreased performance and the rest maintained the same performance. On the second exercise, 3 participants showed improvement, 1 decreased performance and the rest held the same performance. Finally, on the third exercise, 5 participants showed improvement, 2 showed a decrease of performance and the rest kept the same level of performance.

More desirable improvement levels were observed with regards to the memory exercises. For the fourth exercise (first memory exercise), a mean improvement of $6.14 \%$ was observed. For the fifth exercise, the mean improvement was $8.95 \%$. Finally, the sixth exercise showed the highest percentage of improvement, reaching $36.84 \%$.

Individual results are shown in Figure 9 where we can see that for the fourth exercise, 7 participants showed improvement, 3 experienced decreased performance, and the rest kept the same performance. For the fifth exercise, 11 participants displayed improvement, 7 had a decrease of performance, and the rest kept the same performance. Finally, for the sixth exercise, 12 participants showed improvement, 2 had decreased performance and the rest kept the same level of performance.

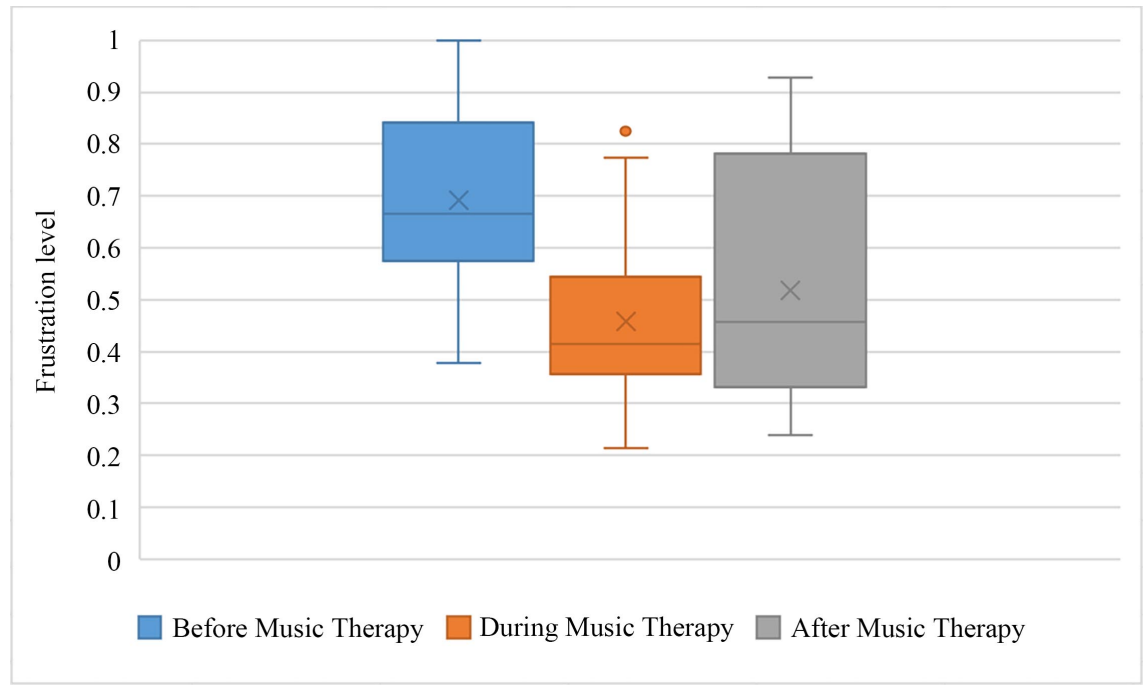

Figure 6. Boxplot of general mean frustration.

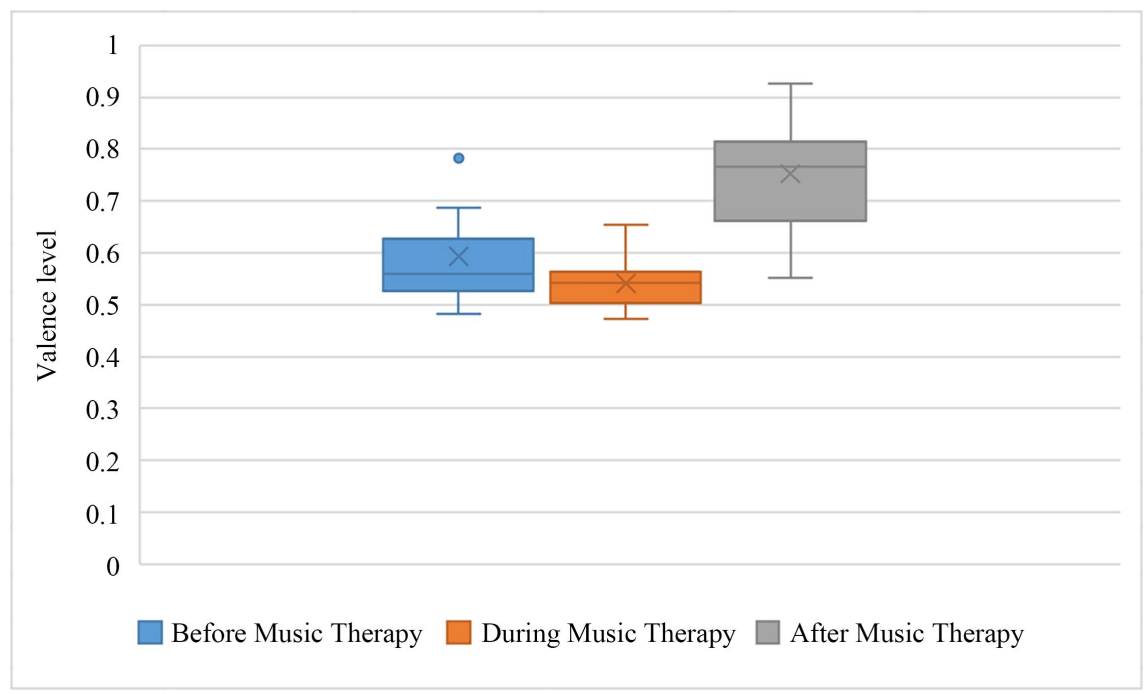

Figure 7. Boxplot of general mean valence. 


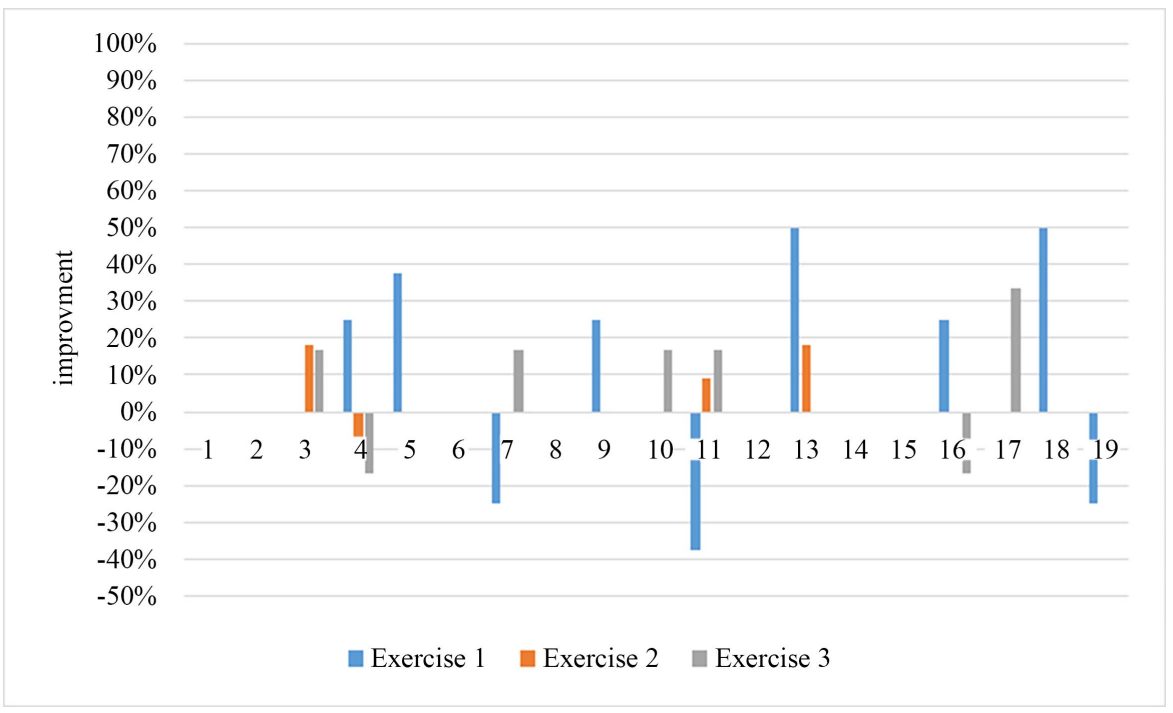

Figure 8. Histogram of performance improvement in attention exercises (1,2 and 3).

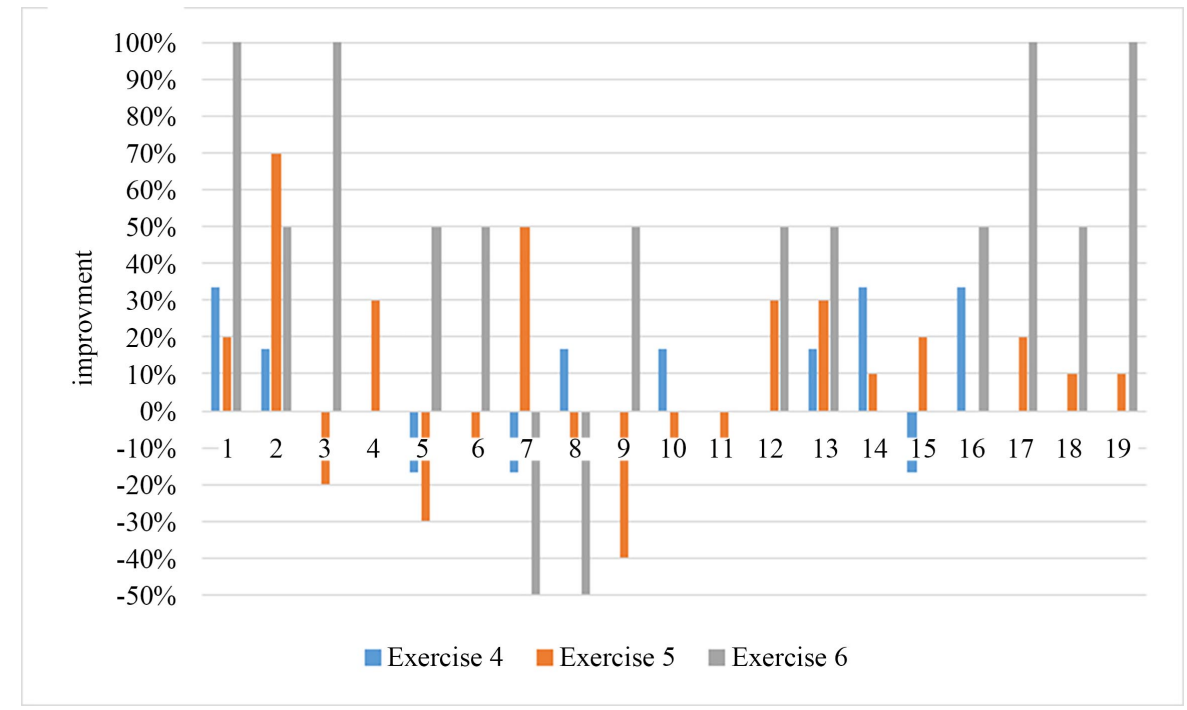

Figure 9. Histogram of performance improvement in memory exercises $(4,5$ and 6$)$.

These results show a considerable increase in memory performance following the adaptive virtual music therapy and only a small improvement in attention abilities.

\section{DISCUSSION}

In this paper, we develop a theory explaining how music therapy can enhance cognitive functions. Our experimental study aimed to identify the emotional and cognitive impact of a virtual music therapy environment on people suffering from subjective cognitive decline (SCD). Results of the study showed that following the intervention, both emotional state and cognitive performance was improved for most participants. Our results are, at first view, in accordance with our hypothesized theory, which was: By increasing positive emotions and reducing negative emotions through the activation of the brain reward system (BRS), music improves cognitive functions. 
The first research question considered that music would activate the BRS and thus influence the emotional state of the participants. As this has already proven to be the case through many studies and was explored in a previous section, it was unsurprising to observe changes in emotional states of participants as they listened to the different songs. In general, negative emotions were reduced, as observed through the measure of frustration, and positive emotions were increased, as seen through the measure of valence. Following the experiment, a large majority of the subjects reported having enjoyed the session. In concordance with the literature $[9,45,46]$, we believe this emotional change is a result of the activation of the BRS.

The second research question asked if the positive change in emotional state would result in better memory and attention performances. Our experimental results show a clear improvement in memory performance for most participants and a small tendency for improvement in attention performance. We propose that these increases in performance are a result of the change in emotional state generated by the music therapy. Although we do not have a direct physiological measurement proving causation from the emotional state to the cognitive state, many studies have shown strong correlations between emotions and mental states. For example, frustration is tightly tied to a high workload, and the inverse is also true. Reduction in workload correlates with reduced frustration [47]. Indeed, we base ourselves on the many studies which have shown correlation between better cognitive states (i.e. less stress, more engagement) and better performance scores on cognitive tasks [48].

Importantly, many findings have established that positive mood states enhance memory function [48]. The theory we put forth merely proposes a process by which music influences memory. We created logical links between music, the BRS and emotions to explain how music influences cognitive functions. Through EEG analysis and cognitive performance assessment, the results of our experiment are in line with our theory.

Our results show that the neurofeedback agent was indeed effective in choosing the appropriate song to play at the end of the intervention. As targeted, negative emotions were reduced, and positive emotions were increased following the virtual experience. The intelligent agent successfully improved emotional states.

Interestingly, the mean valence decreased during the music therapy session, and was followed by an important increase post-session. We believe this is due to how the virtual music therapy session was designed: for the first and longest part timewise, eight different songs are presented, some of which could have given rise to negative emotions. For example, one might have associated "Ave Maria" to the song played at someone's recent funeral, generating negative emotions. The second and final part of the intervention was reserved to play the song which most improved the emotional state of the participant. For this reason, we believe the overall valence during the experienced decreased slightly, and the post-session valence was greatly increased due to the last, optimized song, having the most influence on the post-session emotional states.

Our results show a noticeable difference between the performance improvements of the memory and the attention tasks, with the memory improvements exceeding those of the attention improvements. We propose that this is due to facilitated memory access generated by music. As mentioned in an earlier section of this paper, the amygdala, a structure part of the BRS, is an important player in emotional processing and implicit memory. A study describes how emotional context associated with music and its saliency may lead to preserved residual memory functions in AD. They propose that since music is a salient emotional stimulus, it could potentially facilitate the access to the amygdala-based network and in turn provide better memory functioning [3]. This could explain why our participants experienced a small increase in attention performance and a larger increase in memory performance.

In summary, our experimental study provides support to our theory, which proposed that music helps cognitive functions through the activation of the BRS which provides a better mental environment for cognitive tasks. Future research should investigate through different imaging techniques the activation of the amygdala during music therapy sessions and memory tasks. 


\section{CONCLUDING REMARKS}

The aim of this paper was to introduce and support a theory by which music can enhance cognitive functions in Alzheimer's disease. We propose that the pleasurable experience of music is directly tied to the activation of the brain reward system; a network of interconnected brain structures was involved in dopaminergic activity and in turn the rewarding and motivational experience. The activation of these pathways leads to increased release of dopamine and therefore the subjective experience of pleasure in the listener's brain. This uplift in emotional state can be linked with better cognitive performances, as suggested by various studies in the fields of neuroscience and computer science's intelligent tutoring systems. We explore this theory through a study we conducted on people suffering from subjective cognitive decline. Results show that following the music therapy session, participants had increased positive emotions, reduced negative emotions, slightly increased attention performance and increased memory performance. Supporting our theory, we propose that the cognitive performance increase is a result of a better cognitive environment generated by the music. We propose that by activating the reward system-increasing the dopaminergic release-and in turn generating positive emotions, music can positively affect memory and other cognitive functions.

\section{ACKNOWLEDGEMENTS}

We acknowledge NSERC-CRD (National Science and Engineering Research Council) and Beam Me Up for funding this work.

\section{CONFLICTS OF INTEREST}

The authors declare no conflicts of interest regarding the publication of this paper.

\section{REFERENCES}

1. Dunbar, R.I.M. (2012) On the Evolutionary Function of Song and Dance. In: Bannan, N., Ed., Music, Language, and Human Evolution, Oxford University Press, Oxford, 201-214.

2. Lord, T.R. and Garner, J.E. (1993) Effects of Music on Alzheimer Patients. Perceptual and Motor Skills, 76, 451-455. https://doi.org/10.2466/pms.1993.76.2.451

3. Thaut, M.H. (2010) Neurologic Music Therapy in Cognitive Rehabilitation. Music Perception, 27, 281-285. https://doi.org/10.1525/mp.2010.27.4.281

4. Landes, A.M., Sperry, S.D., Strauss, M.E. and Geldmacher, D.S. (2001) Apathy in Alzheimer's Disease. Journal of the American Geriatrics Society, 49, 1700-1707. https://doi.org/10.1046/j.1532-5415.2001.49282.x

5. Abdessalem, H.B., Byrns, A., Cuesta, M., et al. (2020) Application of Virtual Travel for Alzheimer's Disease. Proceedings of the 9th International Conference on Sensor Networks, Valletta, Malta, 28-29 February 2020, 52-60. https://doi.org/10.5220/0008976700520060

6. Byrns, A., Ben Abdessalem, H., Cuesta, M., et al. (2020) Adaptive Music Therapy for Alzheimer's Disease Using Virtual Reality. In: Vivekanandan, K. and Christos, T., Eds., Intelligent Tutoring Systems, 16th International Conference, ITS 2020, Athens, Greece, 8-12 June 2020, 214-219.

7. García-Betances, R.I., Arredondo Waldmeyer, M.T., Fico, G. and Cabrera-Umpiérrez, M.F. (2015) Corrigendum: A Succinct Overview of Virtual Reality Technology Use in Alzheimer's Disease. Frontiers in Aging Neuroscience, 7, 80. https://doi.org/10.3389/fnagi.2015.00235

8. Gallego, M.G. and García, J.G. (2017) Music Therapy and Alzheimer's Disease: Cognitive, Psychological, and Behavioural Effects. Neurología (English Edition), 32, 300-308. https://doi.org/10.1016/j.nrleng.2015.12.001

9. Blood, A.J. and Zatorre, R.J. (2001) Intensely Pleasurable Responses to Music Correlate with Activity in Brain 
Regions Implicated in Reward and Emotion. Proceedings of the National Academy of Sciences of the United States of America, 98, 11818-11823. https://doi.org/10.1073/pnas.191355898

10. Routtenberg, A. (1978) The Reward System of the Brain. Scientific American, 239, 154-165. https://doi.org/10.1038/scientificamerican1178-154

11. Kandel, E.R., Schwartz, J.H., Jessell, T.M., Siegelbaum, S.A. and Hudspeth, A.J. (2013) Principles of Neural Science. 5th Edition, McGraw-Hill Education, New York.

12. Alzheimer's Association (2017) Alzheimer's Disease Facts and Figures. Alzheimer's \& Dementia, 13, 325-373. https://doi.org/10.1016/j.jalz.2017.02.001

13. Gaugler, J.E., Yu, F., Krichbaum, K. and Wyman, J.F. (2009) Predictors of Nursing Home Admission for Persons with Dementia. Medical Care, 47, 191-198. https://doi.org/10.1097/MLR.0b013e31818457ce

14. Orgeta, V., Tabet, N., Nilforooshan, R. and Howard, R. (2017) Efficacy of Antidepressants for Depression in Alzheimer's Disease, Systematic Review and Meta-Analysis. Journal of Alzheimer's Disease, 58, 725-733. https://doi.org/10.3233/JAD-161247

15. Lawton, M.P., Van Haitsma, K. and Klapper, J. (1996) Observed Affect in Nursing Home Residents with Alzheimer's Disease. The Journals of Gerontology Series B, 51B, 3-14. https://doi.org/10.1093/geronb/51B.1.P3

16. Arnold, S.E., Hyman, B.T., Flory, J., Damasio, A.R. and Van Hoesen, G.W. (1991) The Topographical and Neuroanatomical Distribution of Neurofibrillary Tangles and Neuritic Plaques in the Cerebral Cortex of Patients with Alzheimer's Disease. Cerebral Cortex, 1, 103-116. https://doi.org/10.1093/cercor/1.1.103

17. Brodal, H.P., Osnes, B. and Specht, K. (2017) Listening to Rhythmic Music Reduces Connectivity within the Basal Ganglia and the Reward System. Frontiers in Neuroscience, 11, 153. https://doi.org/10.3389/fnins.2017.00153

18. Trost, W., Frühholz, S., Schön, D., et al. (2014) Getting the Beat, Entrainment of Brain Activity by Musical Rhythm and Pleasantness. Neurolmage, 103, 55-64. https://doi.org/10.1016/j.neuroimage.2014.09.009

19. Phillips-Silver, J. and Trainor, L.J. (2005) Feeling the Beat, Movement Influences Infant Rhythm Perception. Science, 308, 1430. https://doi.org/10.1126/science.1110922

20. Ray, K.D. and Mittelman, M.S. (2017) Music Therapy: A Nonpharmacological Approach to the Care of Agitation and Depressive Symptoms for Nursing Home Residents with Dementia. Dementia, 16, 689-710. https://doi.org/10.1177/1471301215613779

21. King, J., Jones, K., Goldberg, E., et al. (2019) Increased Functional Connectivity after Listening to Favored Music in Adults with Alzheimer Dementia. The Journal of Prevention of Alzheimer's Disease, 6, 56-62.

22. De la Rubia Ortí, J.E., García-Pardo, M.P., Iranzo, C.C., et al. (2018) Does Music Therapy Improve Anxiety and Depression in Alzheimer's Patients? The Journal of Alternative and Complementary Medicine, 24, 33-36. https://doi.org/10.1089/acm.2016.0346

23. De la Rubia Ortí, J.E., Pardo, M.P.G., Benllochi, M., et al. (2019) Music Therapy Decreases Sadness and Increases Happiness in Alzheimer Patients: A Pilot Study. Neuropsychiatry(London), 9, 2013-2020. https://doi.org/10.4172/Neuropsychiatry.1000546

24. Chang, Y., Chu, H., Yang, C., et al. (2015) The Efficacy of Music Therapy for People with Dementia: A Meta-Analysis of Randomised Controlled Trials. Journal of Clinical Nursing, 24, 3425-3440. https://doi.org/10.1111/jocn.12976

25. Zhang, Y., Cai, J., An, L., et al. (2017) Does Music Therapy Enhance Behavioral and Cognitive Function in Elderly Dementia Patients? A Systematic Review and Meta-Analysis. Ageing Research Reviews, 35, 1-11. 
https://doi.org/10.1016/j.arr.2016.12.003

26. Peck, K.J., Girard, T.A., Russo, F.A. and Fiocco, A.J. (2016) Music and Memory in Alzheimer's Disease and the Potential Underlying Mechanisms. Journal of Alzheimer's Disease, 51, 949-959. https://doi.org/10.3233/JAD-150998

27. Kandel, E.R., Schwartz, J.H., Jessell, T.M., et al. (2000) Principles of Neural Science. 4th Edition, McGraw-Hill, New York.

28. Naranjo, C.A., Tremblay, L.K. and Busto, U.E. (2001) The Role of the Brain Reward System in Depression. Progress in Neuro-Psychopharmacology and Biological Psychiatry, 25, 781-823. https://doi.org/10.1016/S0278-5846(01)00156-7

29. Zatorre, R.J. (2003) Music and the Brain. Annals of the New York Academy of Sciences, 999, 4-14. https://doi.org/10.1196/annals.1284.001

30. Juslin, P.N. and Västfjäll, D. (2008) Emotional Responses to Music, the Need to Consider Underlying Mechanisms. Behavioral and Brain Sciences, 31, 559-575. https://doi.org/10.1017/S0140525X08005293

31. Arjmand, H.-A., Hohagen, J., Paton, B. and Rickard, N.S. (2017) Emotional Responses to Music: Shifts in Frontal Brain Asymmetry Mark Periods of Musical Change. Frontiers in Psychology, 8, Article ID: 2044. https://doi.org/10.3389/fpsyg.2017.02044

32. Salimpoor, V.N., Van den Bosch, I., Kovacevic, N., et al. (2013) Interactions between the Nucleus Accumbens and Auditory Cortices Predict Music Reward Value. Science, 340, 216-219. https://doi.org/10.1126/science.1231059

33. Dutcher, J.M. and Creswell, J.D. (2018) The Role of Brain Reward Pathways in Stress Resilience and Health. Neuroscience \& Biobehavioral Reviews, 95, 559-567. https://doi.org/10.1016/j.neubiorev.2018.10.014

34. Ghali, R., Abdessalem, H.B. and Frasson, C. (2017) Improving Intuitive Reasoning through Assistance Strategies in a Virtual Reality Game. The 13th International Flairs Conference, Marco Island, Florida, USA, May 22-24, 382-387.

35. Chaouachi, M., Jraidi, I. and Frasson, C. (2015) Adapting to Learners' Mental States Using a Physiological Computing Approach. The 28 th International Flairs Conference, Hollywood, Florida, USA, May 18-20, 257-262.

36. Aspinall, P., Mavros, P., Coyne, R. and Roe, J. (2015) The Urban Brain: Analysing Outdoor Physical Activity with Mobile EEG. British Journal of Sports Medicine, 49, 272-276. https://doi.org/10.1136/bjsports-2012-091877

37. Maskey, M., Rodgers, J., Ingham, B., et al. (2019) Using Virtual Reality Environments to Augment Cognitive Behavioral Therapy for Fears and Phobias in Autistic Adults. Autism in Adulthood, 1, 134-145.

https://doi.org/10.1089/aut.2018.0019

38. De la Torre-Luque, A., Caparros-Gonzalez, R.A. and Bastard, T., Vico, F.J. and Buela-Casal, G. (2017) Acute Stress Recovery through Listening to Melomics Relaxing Music: A Randomized Controlled Trial. Nordic Journal of Music Therapy, 26, 124-141. https://doi.org/10.1080/08098131.2015.1131186

39. Rathbone, C.J., Moulin, C.J. and Conway, M.A. (2008) Self-Centered Memories: The Reminiscence Bump and the Self. Memory \& Cognition, 36, 1403-1414. https://doi.org/10.3758/MC.36.8.1403

40. Valdez, P. and Mehrabian, A. (1994) Effects of Color on Emotions. Journal of Experimental Psychology, General, 123, 394-409. https://doi.org/10.1037/0096-3445.123.4.394

41. Sherlin, L.H., Arns, M., Lubar, J., et al. (2011) Neurofeedback and Basic Learning Theory: Implications for Research and Practice. Journal of Neurotherapy, 15, 292-304. https://doi.org/10.1080/10874208.2011.623089

42. Watson, D., Clark, L.A. and Tellegen, A. (1988) Development and Validation of Brief Measures of Positive and Negative Affect: The PANAS Scales. Journal of Personality and Social Psychology, 54, 1063-1070.

https://doi.org/10.1037/0022-3514.54.6.1063 
43. Kennedy, R.S., Lane, N.E., Berbaum, K.S. and Lilienthal, M.G. (1993) Simulator Sickness Questionnaire: An Enhanced Method for Quantifying Simulator Sickness. The International Journal of Aviation Psychology, 3, $203-220$. https://doi.org/10.1207/s15327108ijap0303_3

44. Lallemand, C., Koenig, V., Gronier, G. and Martin, R. (2015) Création et validation d'une version française du questionnaire AttrakDiff pour l'évaluation de l'expérience utilisateur des systèmes interactifs. European Review of Applied Psychology, 65, 239-252. https://doi.org/10.1016/j.erap.2015.08.002

45. Brown, S., Martinez, M.J. and Parsons, L.M. (2004) Passive Music Listening Spontaneously Engages Limbic and Paralimbic Systems. NeuroReport, 15, 2033-2037. https://doi.org/10.1097/00001756-200409150-00008

46. Menon, V. and Levitin, D.J. (2005) The Rewards of Music Listening: Response and Physiological Connectivity of the Mesolimbic System. Neurolmage, 28, 175-184. https://doi.org/10.1016/j.neuroimage.2005.05.053

47. Chaouachi, M. and Frasson, C. (2012) Mental Workload, Engagement and Emotions: An Exploratory Study for Intelligent Tutoring Systems. In: Cerri, S.A., Clancey, W.J., Papadourakis, G. and Panourgia, K., Eds., Intelligent Tutoring Systems, ITS 2012, Lecture Notes in Computer Science, Springer, Berlin, 65-71. https://doi.org/10.1007/978-3-642-30950-2_9

48. Jensen, U., Lewsi, B., Tranel, D. and Adolphs, R. (2004) Emotion Enhances Long-Term Declarative Memory. Proceedings of the Society for Neuroscience, 203-209. 